\title{
Simulation of heat distribution in particles during micro field-activated sintering by considering electrical concentration and contact resistance
}

\author{
Dan Zhao ${ }^{1}$, Jie Wang ${ }^{1, a}$, Yi Yang ${ }^{1}$, Gang Yang ${ }^{1}$, Yi Qin², Jian Liu ${ }^{1}$, and Deqiang Yin ${ }^{1}$ \\ ${ }^{1}$ School of Manufacturing Science and Engineering, Sichuan University, Chengdu, Sichuan 610065, \\ PR China \\ ${ }^{2}$ Department of Design, Manufacture and Engineering Management, University of Strathclyde, \\ James Weir Building. 75 Montrose Street, Glasgow G1 1XQ, UK
}

\begin{abstract}
Finite element simulations have been conducted to investigate the heat distribution between the particles from contact resistance and current density during the Micro-forming Fields Activated Sintering Technology (Micro-FAST). Results of effect for the arrangement modes of powder particles (Series connection and parallel connection) showed that the electro-heat focusing could be attributed to the remarkable difference of contact resistance between the particles.
\end{abstract}

\section{Introduction}

With the development of microminiaturization in manufacturing, the demand of micro-parts is increased. The preparation methods which can prepare micro-parts with excellent properties and high accuracy attract researchers' attention. Recently, Yi Yang et al. proposed a new manufacturing technology named as Micro-forming Fields Activated Sintering Technology (Micro-FAST) for the preparation of microparts $[1,2]$. Figure 1 shows the schematic of Micro-FAST. Loose powders were directly placed into a die, and then heated to the sintering temperature by passing an AC current through the die, and pressure is applied onto the powders at the same time.

Micro-gears with eight teeth and $1.6 \mathrm{~mm}$ pitch diameter were successfully prepared by Micro-FAST, shown in Fig. 2. A relative density of $98.7 \%$ was achieved [3]. Micro-parts can be prepared in a short time (about $10 \mathrm{~min}$ ) with a high relative density (over 90\%) through Micro-FAST, indicating that MicroFAST is more effective way in comparison with the traditional sintering technology. The previous study shows that plastic deformation of particles and the formation of liquid phase are two main factors for the densification of samples. The formation of liquid phase and the plastic deformation are both influenced by temperature. So the research on the heat distribution of samples during Micro-FAST is quite meaningful to the research of densification mechanism.

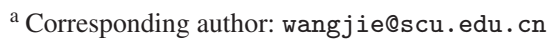

This is an Open Access article distributed under the terms of the Creative Commons Attribution License 4.0, which permits unrestricted use, distribution, and reproduction in any medium, provided the original work is properly cited. 


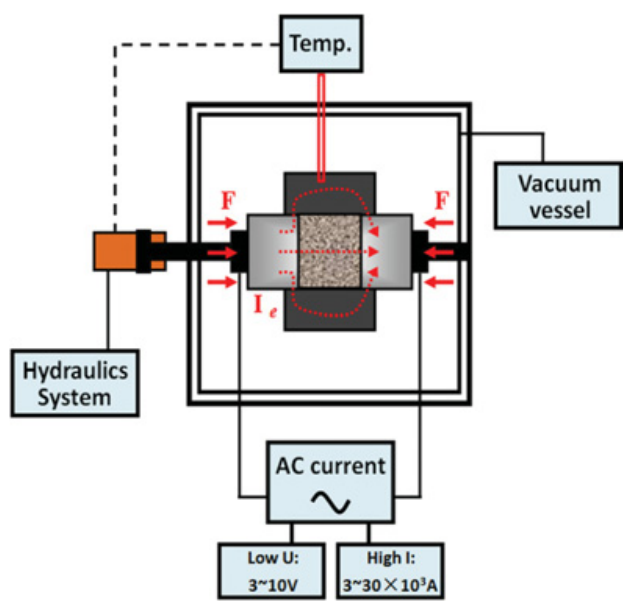

Figure 1. The schematic of Micro-Fields Activated Sintering.

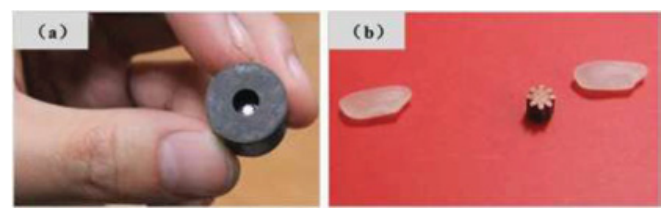

Figure 2. Micro gears prepared by Micro-FAST.

The accurate measurement of heat is quite difficult during the Micro-FAST, especially in comparing the different sintering cases. However, Finite Element Method (FEM) is one of the important computational mechanics, started from the middle of the 20th century. It is well known that the FEM plays a large role in assistant analysis, assistant design and product prediction, particularly in multi-fields activated sintering, that is to say, FEM has been widely used for the simulation of physical problems in electromagnetism, thermology and engineering mechanics [4-6]. For instance, K. Vanmeensel et al. [7] studied the evolution of current density and heat distribution during field activated sintering by finite element calculations. And, S. Munoz U. Anselmi-Tambrrini [8] analysed the various experimental parameters by finite element method, and evidenced their role in determining the heat distribution during field activated sintering. Furthermore, X. Wang et al. [9] used a finite element model to simulate threeway coupling of heat, electrical and mechanical behaviour of electric current activated sintering. As well, J. Zhang et al. [10] discussed the effect of the electric current pattern on heat distributions in the compacted powders, and the effect of compaction modes on conductivity in spark plasma sintering by using the discrete finite-element method. Moreover, C. Wang et al. [11] analysed the heat and stress distribution in spark plasma sintering through a heat-electrical-mechanical coupled and dynamic finite element model. All these studies proved that FEM is a reliable method to simulate heat distribution.

In this paper, based on the experimental data for the preparation of micro-gears, simplified models about sintering body were built by FEM to simulate the heat distribution and investigate the influences of contact resistance on heat distribution. 


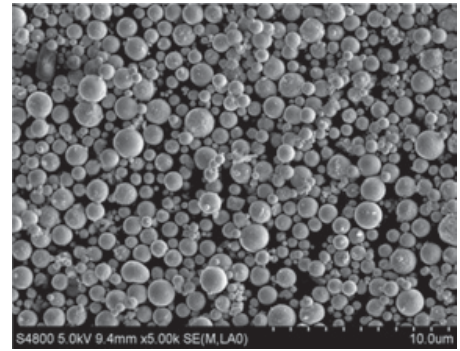

Figure 3. An SEM image of original powders.

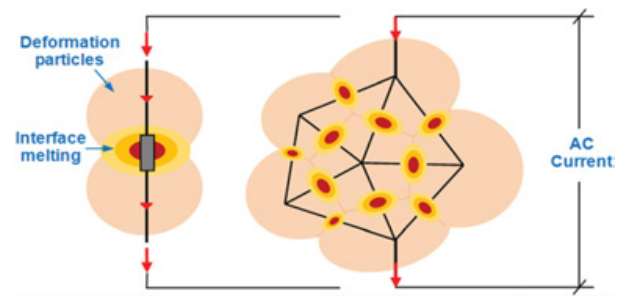

Figure 4. The schematic of circuits formed during Micro-FAST.

\section{Finite element modelling}

Different from heat conduction in traditional sintering, samples was heated by Joule heat generated by electric current during Micro-FAST. When electric current goes through the sintering materials, resistance heating will be produced quickly. According to the Joule-Lenz's law, the heat generated by the electric current can be given by

$$
Q=I^{2} R t
$$

where $Q$ is the heat generated by the current passing through the powders, $I$ the electric current, $R$ the resistance of the particle or the contact resistance, and $t$ the time. When sintering time and the current density is fixed, the heat is only related to the resistance, that is to say, the resistance would greatly influence the heat distribution.

Materials sintered are loose powders with regular spherical particles, as shown in Fig. 3. The sizes of spherical particles are not uniform, and the gap exists among the particles. Moreover, the particles would be in contact each other under pressure during the sintering. All of these will lead to the uneven resistance in different areas within the sintering body and generate uneven distribution of Joule heat.

According to the condition of sintering materials, series connection and parallel connection both exist during the sintering, as shown in Fig. 4.

In order to simplify the calculation, four spherical models were selected for the finite element modeling, as shown in Fig. 5. Figure 5(a) is the series connection and Fig. 5(b) the parallel connection. The radius of all the spherical models was set as $15 \mu \mathrm{m}$, in accorance with the average size of sintering powders. And the distance between two centers of sphere was set to $29.5 \mu \mathrm{m}$ (less than the diameter of spherical $30 \mu \mathrm{m}$ ) to represent the contact. Series connection and parallel connection cases were investigated, respectively.

Element type was selected as PLANE67. In material props, heat unit was set as Celsius, while electromag unit was selected as MKS system. Two material models were built for the particle part and contact part. The model for the particle part was regarded as Model 1; the model for the contact part 
(a)

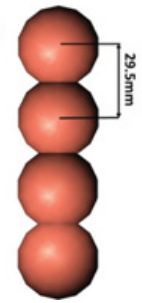

(b)

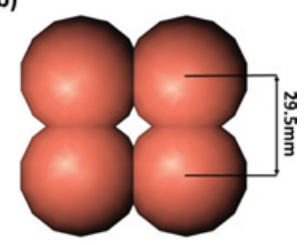

Figure 5. Finite element modeling: (a) series connection; (b) parallel connection.
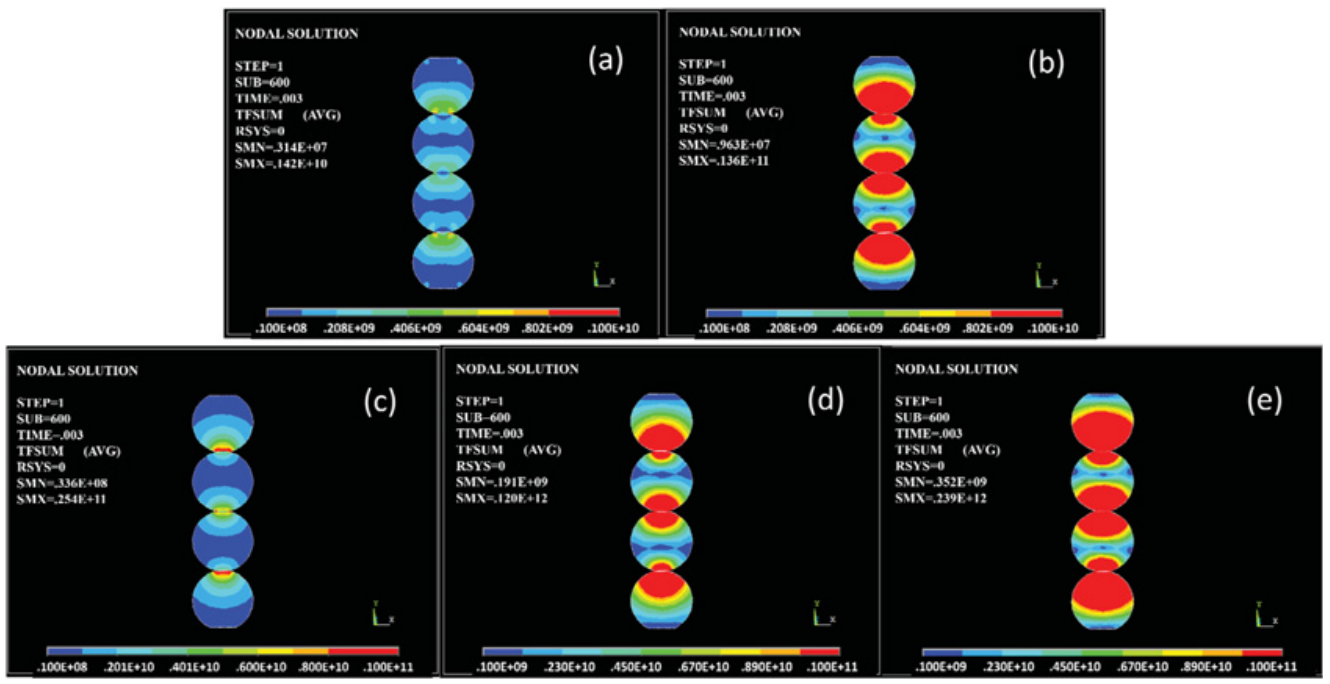

Figure 6. Simulation results of heat distributions with fixed input current (1A) and varing contact resistances in series connection: (a) $\times 1$; (b) $\times 50$; (c) $\times 100$; (d) $\times 500$; (e) $\times 1000$.

was regarded as Model 2. Materials for two models were set as copper. The resistance was the only difference between two models.

Controlling variables method was used to analyze the main cause for the heat distribution during Micro-FAST. The same analyzing method was used in series connection and parallel connection. Firstly, the resistances of two models were set as equal $\left(\mathrm{T}=0^{\circ} \mathrm{C}, \mathrm{R}=1.6 \mathrm{E}-14 \mathrm{~T} \Omega \cdot \mu \mathrm{m} ; \mathrm{T}=20^{\circ} \mathrm{C}\right.$, $\mathrm{R}=1.75 \mathrm{E}-14 \mathrm{~T} \Omega \cdot \mu \mathrm{m})$. Then set a given electric current (1A) to simulate the heat distribution. Next, fix the value of electric current, and change the resistance value of model 2 to inveatigate the influence of contact resistance on the heat distribution.

\section{Results and analysis}

\subsection{Simulation results of series connection}

In series connection, the simulated results of heat distribution with fixed input-current (1A) and various contact resistances are shown in Fig. 6. It can be seen that in Fig. 6(a), the value of contact resistance is equal to the particle resistance. The contact resistances are set as 50 times, 100times, 500times and 1000times the particle resistance in Fig. 6(b), (c), (d) and (e), respectively. As can be seen in Fig. 6, the heat distributions under different contact resistances are symmetrical. Furthermore, it can be found that the higher heat is presented at the contact parts, and the lower heat is presented at the parts far 

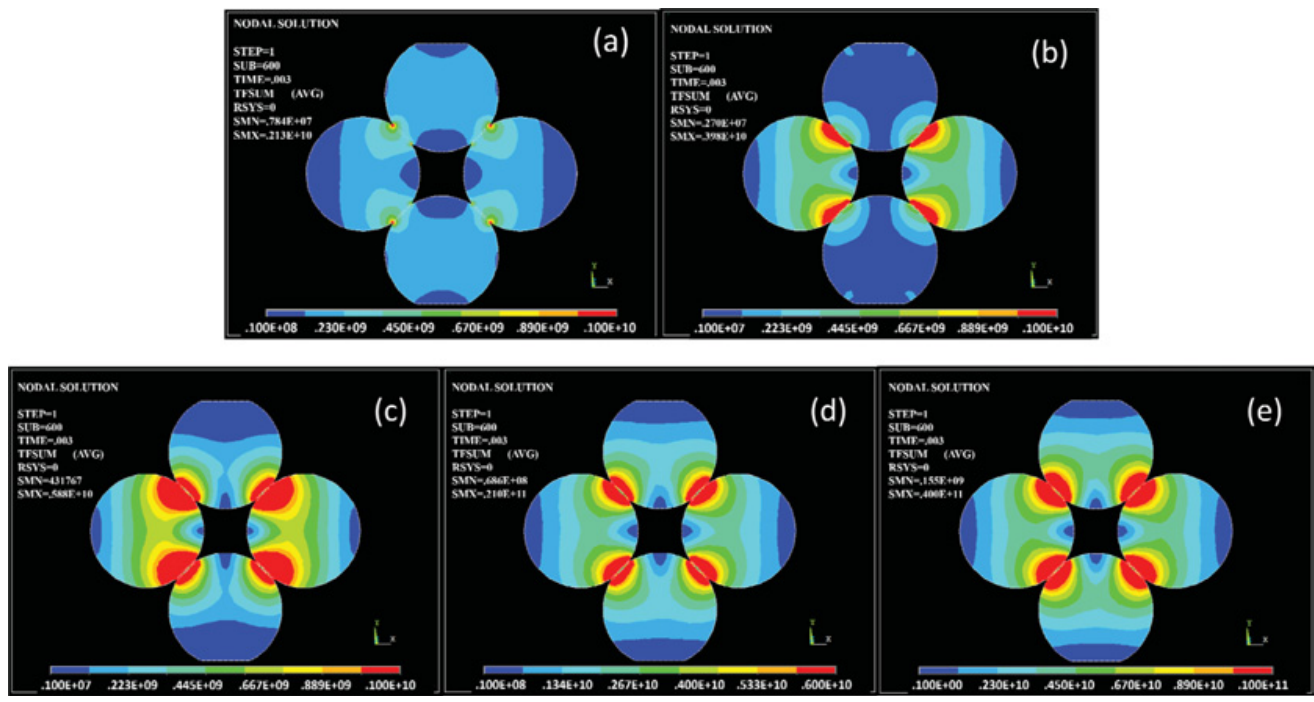

Figure 7. Simulation results of heat distributions with fixed input current (1A) and varing contact resistances in parallel connection: (a) $\times 1$; (b) $\times 50$; (c) $\times 100$; (d) $\times 500$; (e) $\times 1000$.

away from the contact. The high heat area is expanded with the increasing contact resistance. And the maximum values of generated heat in serise connection are increased from $0.142 \mathrm{e} 10$ to $0.136 \mathrm{e} 11$, $0.254 \mathrm{e} 11,0.120 \mathrm{e} 12$ and $0.239 \mathrm{e} 12$, corresponding to the increased contact resistance from 1 time to 50 times, 100 times, 500 times and 1000 times. It is worth noting that the values in present study are only relative significance.

\subsection{Simulation results in parallel connection}

In parallel connection, the simulated results of heat distribution with fixed input-current (1A) and various contact resistances are shown in Fig. 7. Not surporisingly, the value of contact resistance is equal to the particle resistance in Fig. 7(a), similar to the series connection. The contact resistances are set as 50 times, 100 times, 500 times and 1000 times the particle resistance in Fig. 7(b), (c), (d) and (e), respectively. The heat distributions under different contact resistances are also symmetrical in parallel connection. With the increasing contact resistance, the generated heat has increased and its distribution is more symmetrical. On the whole, the high heat is presented at the contact parts, and the low heat is presented at the parts far away from the contact, which is also similar to the series connection. The maximum values of the generated heat in parallel connection are increased from $0.213 \mathrm{e} 10$ to $0.398 \mathrm{e} 10$, $0.588 \mathrm{e} 10,0.210 \mathrm{e} 11$ and $0.400 \mathrm{e} 11$, corresponding to the increased contact resistance from 1 time to 50 times, 100 times, 500 times and 1000 times.

\subsection{Analyses}

The heat distributions in both series connection and parallel connection, are symmetrically but nonuniformly distributed with the fixed input current and varing contact resistances. When the contact resistance is equal to the particle resistance, the heat focusing in the contact area, as shown in Fig. 6(a) and Fig. 7(a), are generated by focusing current density. The heat in connections is generated by electric current, according to Joule-Lenz's law. So when the contact resistance is increased, the heat of contact 


\section{MATEC Web of Conferences}

area is increased as well. As a result, the electro-heat focusing is presented obviously. And the high heat produced by the high contact resistance would influence the around area, expanded the high heat area.

By comparing the maximum values of heat in two kinds of connections, the maximum value of heat in series connection is higher than that in parallel connection at the same current and resistance, which could be attributed to the shunting effect in parallel connection. Besides, the high heat area in series connection is also larger than that in parallel connection. It can be concluded that series connection is more feasible to generate high heat and expand the high heat area, where the applied pressure plays the role in increasing the contacting area and changes the connectting situation between the particles.

\section{Conclusions}

From the work completed in this study, the following conclusions can be drawn:

(1) Heat generated during Micro-FAST is nonuniform but symmetrically distributed. High heat is presented in contact parts, causing electro-heat focusing.

(2) Contact resistance is one of the reasons to cause electro-heat focusing. Electro-heat focusing is more obviously presented in higher contact resistance.

(3) The maximum value of heat and high heat area are much larger in series connection than that in parallel connection.

This work was funded in part by the National Nature Science Foundation of China (No. 51275322), the UK Royal Society/ China NSFC International Exchanges Programme (No. 51311130134).

\section{References}

[1] A.K. Du, Y. Yang, Y. Qin, G. Yang, Powder Metal. Met. Ceram., 52, 261 (2013)

[2] K.L. Huang, Y. Yang, Y. Qin, G. Yang, Mater. Manuf. Process., 28, 183 (2013)

[3] D. Lu, Y. Yang, Y. Qin, G. Yang, J. Microelectromech. S., 22, 708 (2013)

[4] G.F. Wei, X.D. Yu, Q.S. Ren, Biomed. Eng.-Appl., Bas. C., 27, 1550010 (2015)

[5] C. Renno, M.D. Giacomo, Energies, 7, 7395 (2014)

[6] J. Donea, S. Giuliani, J.P. Halleux, Comput. Method. Appl. M., 33, 689 (1982)

[7] K. Vanmeensel, A. Laptev, J. Hennicke, J. Vleugels, O. Van der Biest, Acta Mater., 53, 4379 (2005)

[8] S.M. Anselmi-Tamburini, Int. J. Manuf. Technol., 65, 127 (2013)

[9] X. Wang, S.R. Casolco, G. Xu, J.E. Garay, Acta Mater., 55, 3611 (2007)

[10] J. Zhang, A. Zavaliangos, J. Electron. Mater.,4, 873 (2011)

[11] C. Wang, L.F. Cheng, Z. Zhao, Comp. Mater. Sci., 49, 351 (2010) 